\title{
ELECTRON-POSITRON PAIR WINDS FROM CENTRAL LUMINOUS ACCRETION DISK WITH RADIATION DRAG
}

\author{
Y. TAJIMA AND J. FUKUE \\ Astronomical Institute, Osaka Kyoiku University \\ Asahigaoka, Kashiwara, Osaka 582, Japan
}

The accretion disks are now supposed to be the main driving source of the active astrophysical phenomena. Even the electron-positron pair plasma will be created at the surface of the sufficiently luminous disk. While the effect of radiation drag which causes in the intense radiation fields around the accretion disk is examined recently. Then, we numerically consider the radiative accelerated pair-winds, which blow off from central luminous accretion disk surrounding a black hole, taking into account radiation drag of the order of $v / c$.

\section{Disk Radiation Fields}

For the present purpose, we quantitatively calculate the full components of radiation fields produced by the standard accretion disk around a black hole, considering the Doppler enhancement to the order of $v / c$ (Tajima and Fukue 1997). We emphasize that there appears the azimuthal component of radiative flux, since the disk radiation field has an angular momentum.
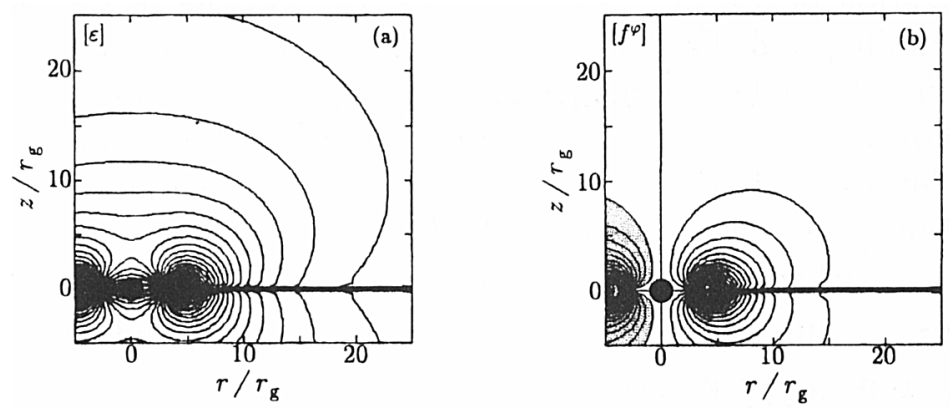

Figure 1. Examples of the contour maps of radiation fields around the accretion disk. (a) The normalized radiation energy density $\varepsilon$. (b) The azimuthal component of normalized radiative flux $f^{\varphi}$. 


\section{Motions of Pair Winds}

Here, we assume that the electron-positron pair plasma is created at an inner region of the disk, and the pair winds blow off explosively from there. Using the components of the radiation fields, we examine the accretion disk winds which are ejected from the central region of the luminous disk. From the comparison between the winds from the less luminous (Fig.2a) and the luminous (Fig.2b) disks, and the non-dragged winds (Fig.2c), it can be understood that the winds, which blow off from luminous disks, are collimated in the vertical direction. Moreover, the radiation drag also collimates the winds. The collimation occurs since the angular momentum of the winds is removed by the radiation drag rapidly (Fig.3). However it is difficult for the streamlines to be collimated to the very close to the $z$-axis only by the radiation drag, due to the radial component of the radiation flux. Thus, the winds appear the conical streamlines.
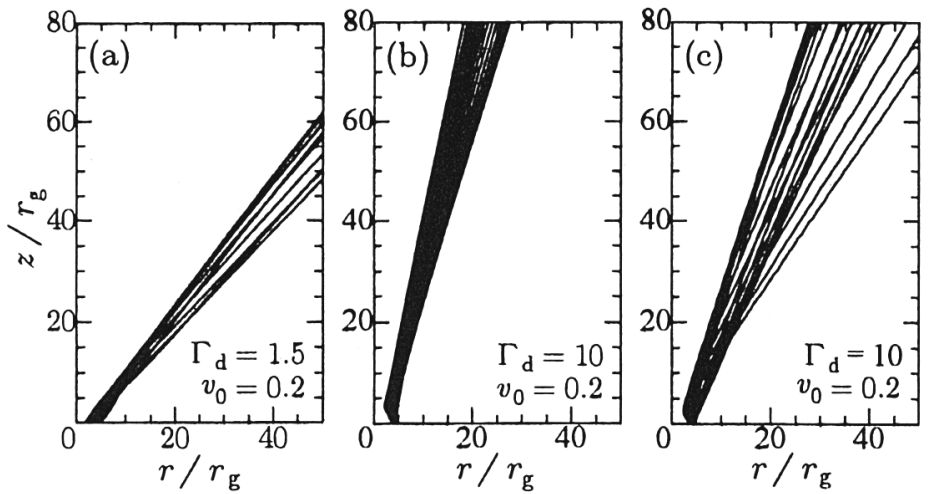

Figure 2. Examples of trajectories of the winds. The parameter $\Gamma_{d}$ is the disk luminosity normalized by Eddington luminosity, and $v_{0}$ is the initial velocity. (a) Dragged winds from a low luminous disk. (b) Dragged winds from a luminous disk. (c) Non-Dragged winds from a luminous disk.

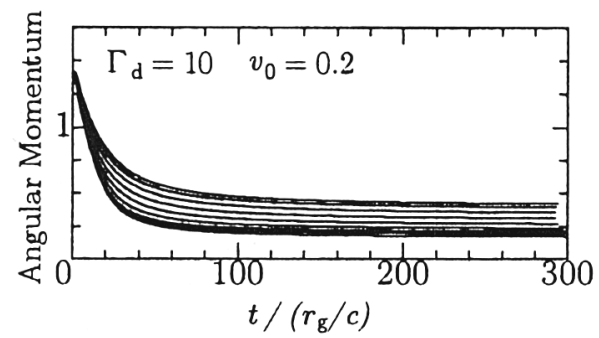

Figure 3. The time variation of the angular momentum of the dragged winds.

\section{References}

Tajima Y., Fukue J. (1997) Radiative Disk Winds under Radiation Drag II, PASJ, submitted 